ADULTHOOD

Age trends in musical preferences in adulthood: 2 . Sources of social influences as determinants of preferences

Date: 04 March 2017 
ADULTHOOD

\title{
Age trends in musical preferences in adulthood: 2 . Sources of social influences as determinants of preferences
}

\begin{abstract}
Using the Music Preferences in Adulthood Model (MPAM, Bonneville-Roussy et al., 2017), an integrative model of the psychological determinants of musical preferences in adulthood, the main objective of this paper is to investigate how different sources of social influences may affect musical preferences in adulthood. More specifically, we examine how the social network (such as family and peers), and psychological interpersonal dispositions (such as one's psychological tendencies towards conformity) may influence these age trends. In two studies with a total sample size of over 950 adults, we examine how social influence variables are associated with age trends in musical preferences, measured with music genres and music clips (samples of musical audio recordings). Results revealed that the social network and interpersonal dispositions are related to preferences for different musical dimensions. These relationships were stronger for the musical genres dimensions as compared with the musical clips dimensions. In addition, our findings show that dispositions toward conformity moderated the relations between age and musical preferences in adulthood. Those results confirm that social influences, especially the interpersonal disposition towards conformity, are important in the adoption of different musical preferences as adults evolve in age. The results are discussed within a lifespan developmental psychology of music perspective.
\end{abstract}

Keywords: Musical preferences, age differences, adulthood, social influences, Music preferences in adulthood model 
ADULTHOOD

\section{Age trends in musical preferences in adulthood: 2. Sources of social influences as determinants of preferences}

Musical preferences, or musical taste, are undeniably social. In adolescence, music serves as a badge for identity and group formation (Arnett, 1992, 1995;

Hargreaves, North, \& Tarrant, 2006; Macdonald, Hargreaves, \& Miell, 2002; North \& Hargreaves, 1999). The development of musical preferences in adulthood has also been linked with social functions: As individuals age, their social roles changes and those roles may be better aligned with different music styles (Bonneville-Roussy, Rentfrow, Xu, \& Potter, 2013). The social function of music in adolescence has interested researchers for decades (see Hargreaves et al., 2006), however little is understood about how musical preferences are subject to different sources of social influences in adulthood. Recently, Schäfer (2016) and Schäfer and colleagues (2016) have found that the musical taste of adults varied according to the function they attributed to music. The researchers noted that social relatedness was mentioned as a source of musical preferences (Schäfer, 2016). In addition, positive and negative social feedback successfully modified the strengths of musical preferences of adults in an experimental setting (Schäfer et al., 2016). Clearly, further research is needed to evaluate the role of social influences in the musical taste of adults. This paper is the second of a series of three papers that aim to provide a preliminary understanding of the psychological determinants of age trends in musical preferences in adulthood.

In a first of a series of papers aimed at exploring the determinants of musical preferences in adulthood, we presented the Music Preferences in Adulthood Model (MPAM) that seeks to explain the psychological determinants of age differences in 
ADULTHOOD

musical preferences in adulthood (Bonneville-Roussy, Stillwell, Kosinski, \& Rust, 2017).

With a sample of more than 4,000 adults, we have found that age trends in musical taste were relatively similar when measured with music genres and with extract of audiorecordings (music clips). The age trends revealed in this investigation mostly replicated the trends found in past research (Bonneville-Roussy et al., 2013), confirming the validity of the age trends, and broadly speaking, the assumption that musical preferences might not be fixed in adulthood (Bonneville-Roussy et al., 2013, 2017; Greasley \& Lamont, 2006; Hargreaves \& Bonneville-Roussy, 2017; Hemming, 2013; North, 2010). In a nutshell, Bonneville-Roussy et al. (2017) have found three main age trends in musical preferences: An upward trend composed of the Jazzy and Unpretentious dimensions that were increasingly liked with age, a stable trend with the Classical dimension, and a downward trend, composed of the Contemporary and Intense dimensions, that were gradually more disliked with age.

In addition, MPAM posits that age differences in musical preferences in adulthood may be determined by three intrinsic and extrinsic psychological determinants. Two of those determinants, individual differences and social influences, pertain to the individuals who listen to music, and are therefore considered as extrinsic to the music. Individual differences in musical preferences have been the subject of a vast literature, with concepts such as gender differences, demographic differences and differences in personality, being examined thoroughly (Bonneville-Roussy et al., 2013; Knox \& MacDonald, 2016; North \& Hargreaves, 2007a, b, c; Rentfrow \& McDonald, 2009). The second extrinsic psychological determinant posited in the MPAM is the various sources of social influences (North \& Hargreaves, 2008b), which has so far been overlooked from 
ADULTHOOD

recent research using samples of adults. The sources of social influences in musical taste in adulthood are the subject of the present investigation. The third determinant, the perceived intrinsic attributes of the music, will be examined in the third paper of the series.

The present work seeks to extend and validate the MPAM (Bonneville-Roussy et al., 2017) by examining some social-psychological determinants of age trends in musical preferences in adulthood. It also examines the moderating roles of those variables on age trends in musical preferences, using the MPAM. In the MPAM, presented in Figure 1, we posit that musical preferences vary with age and that the determinants of musical preferences may act as moderators of the age trends.

Insert Figure 1 about here

\section{Individual differences in age trends in musical preferences in adulthood}

The MPAM (Bonneville-Roussy et al., 2017, see also Figure 1) posits that the first extrinsic determinant of musical taste in adulthood is individual differences. These individual characteristics have been repeatedly related to musical preferences in adulthood in past research: age, gender, ethnicity, personality, education and music education (e.g. Bonneville-Roussy et al., 2013; Greenberg et al., 2016; Greenberg, Monteiro, \& Rentfrow, 2014; North \& Hargreaves, 2007). Their roles in the musical taste of adults have been substantiated many times. As such, this section provides a brief overview of the research on individual differences to date, but since a large amount of evidence already exists in those areas, individual differences are not included in the present empirical investigation. 
ADULTHOOD

Considerable evidence has shown that gender is related to musical preferences.

Broadly speaking, research has shown that women tend to prefer softer music, such as classical, pop and soul (Colley, 2008; Finnäs, 1989; McCown, Keiser, Mulhearn, \& Williamson, 1997; Nater, Abbruzzese, Krebs, \& Ehlert, 2006; North \& Hargreaves, 2007a, 2007b; Schwartz \& Fouts, 2003). On the other hand, men tend to prefer sonically heavier and more forceful types of music with pronounced bass, such as heavy metal and rock (McCown et al., 1997; Nater et al., 2006). This gender difference tends to be present early in adolescence (Delsing, ter Bogt, Engels, \& Meeus, 2008; Hargreaves, Comber, \& Colley, 1995; Mulder, Ter Bogt, Raaijmakers, Nic Gabhainn, \& Sikkema, 2009) and to persist throughout adulthood (North \& Hargreaves, 2007a). In terms of culture and ethnicity, musical preferences in general have been assessed with samples in cultures coming from all continents (Brown, 2012; Chamorro-Premuzic, Goma -i-Freixanet, Furnham, Muro, \& Gomà-i-Freixanet, 2009; Chamorro-Premuzic, Swami, Furnham, \& Maakip, 2009; Delsing et al., 2008; Getz, Chamorro-Premuzic, Roy, \& Devroop, 2011; Langmeyer, Guglhör-Rudan, \& Tarnai, 2012; Miranda \& Claes, 2004; North \& Hargreaves, 2007a; Rentfrow, Goldberg, \& Levitin, 2011; Schäfer \& Sedlmeier, 2010; Ter Bogt, Mulder, Raaijmakers, \& Nic Gabhainn, 2010; Zweigenhaft, 2008). From these studies, it appears that musical preferences have a similar structure between cultures, with some music considered more or less sophisticated, and other music considered more intense within cultures (Miranda, Blais-Rochette, Vaugon, Osman, \& Arias-Valenzuela, 2015; ter Bogt et al., 2012). Scholars have associated higher levels of education and socioeconomic status with taste for more 'high brow', or sophisticated, types of music and 'low brow', or simpler-sounding music. People who prefer 'high brow' music, such 
ADULTHOOD

as classical and jazz, have been found to be comparatively more likely to have obtained an undergraduate degree, to have been to graduate school, and to have higher income (Han, 2003; Katz-Gerro, 1999; Katz-Gerro, Raz, \& Yaish, 2007; Mark, 1998; van Eijck, 2001) The opposite results have been found for 'low brow' music. Finally, personality traits have been associated with musical taste numerous times, with each personality dimensions being related to specific musical preferences dimensions (see, for recent research in the area, Bonneville-Roussy et al., 2013; Greenberg et al., 2016; Knox \& MacDonald, 2016; Langmeyer et al., 2012; Rentfrow, 2012; Rentfrow et al., 2012). However, using a very large sample size of over 30,000 adults, North (2010) have found that overall, personality is a less important predictor of musical preferences than age.

In terms of the moderators of age trends in musical preferences in adulthood, Bonneville-Roussy et al. (2013) have found that the age trends in musical preferences were not moderated by personality traits, or by gender. These result suggest that, since individual differences are relatively stable traits in adulthood, they may not act as moderating variables to explain the various age trends in musical taste found in past research (Bonneville-Roussy et al., 2013, 2017).

\section{The role of the social network and interpersonal dispositions in age trends in musical preferences in adulthood}

Much less is known about the social sources of influences in musical preferences in adulthood. Taking a psychological perspective, two major sources of social influences are important when examining the psychological determinants of age trends in musical preferences. The first source consists of the interpersonal psychological dispositions 
ADULTHOOD

towards conforming to the social standards, or wanting to diverge from them. The second source is the social network of individuals that mostly consists of their groups of peers, colleagues and friends who evolve in similar social environments, as well as family.

Musical preferences can be influenced by individuals' own susceptibility to being influenced by others in their everyday choices, that is, their interpersonal dispositions. Interpersonal dispositions are defined as the psychological tendencies of an individual to behave in a socially predictable manner (Buss \& Craik, 1981). Concepts such as compliance, conformity, and self-awareness have been examined in past music psychology research (Berns, Capra, Moore, \& Noussair, 2010; Egermann, Kopiez, \& Altenmüller, 2013; North \& Hargreaves, 1999, 2008a; Schäfer et al., 2016; Ziv, 2016). This line of research offers great insight in the mechanisms through which musical preferences could be influenced by interpersonal dispositions, but has mostly examined narrow age ranges (mostly adolescents and emerging adults), and therefore offers limited insights about the mechanisms through which those dispositions could influence the musical taste of adults as they evolve in age.

Some social-psychological mechanisms that affect taste have been examined in the broader fashion and taste for goods literature. According to social-psychological research on physical appearance and fashion clothing (Bearden \& Rose, 1990; Martin, Leary, \& Rejeski, 2000), individuals who have the tendency to monitor their behaviour when they manage their self-presentation tend to prefer buying goods that are fashionable and mainstream. This research has shown that conformity not only influences the adoption of fashion, but also forces the abandonment of fashion (Berger \& Heath, 2008). People who seek individuality tend to prefer goods that are unique and less mainstream 
ADULTHOOD

(He, Cong, Liu, \& Zhou, 2010). Since individuals often display their group membership

and social status via musical taste (Bakagiannis \& Tarrant, 2006; Bennett \& Taylor, 2012; Hays \& Minichiello, 2005; Rentfrow \& Gosling, 2006), it is likely that adults are subject to similar social influences for fashion and music. In adulthood, individuals use their musical taste to negotiate social situations, differentiate themselves from dissimilar others and synchronise themselves to similar others (Harrison \& Ryan, 2010). A growing body of research has shown that adults use music to form impressions of others in zeroacquaintance situations, and that adolescents tend to form more stable friendships with peers who share the same musical preferences as themselves (Boer et al., 2011; Rentfrow \& Gosling, 2006; Selfhout, Branje, ter Bogt, \& Meeus, 2009).

Conformity, self-monitoring and uniqueness seeking are three of the interpersonal disposition processes through which individuals may use information conveyed by music to manage their self-presentation (Leary \& Allen, 2011). Only a handful of studies have examined the links between interpersonal disposition and musical preferences (see recent research and reviews by North \& Hargreaves, 2008b; Schäfer et al., 2016; Ziv, 2016). A series of independent studies reported in Finnäs (1989) and North \& Hargreaves (1999) has shown that children and adolescents are far more likely to modify their preferred music choice if they know that this choice will be disclosed to peers, as they want to conform to the preferences of those peers. Conformity can thus lead adolescents to adopt different musical preferences to avoid negative social consequences. This association has not yet been investigated in adulthood. Self-monitoring is the tendency of individuals to adapt their behaviour in social situations to gain or maintain social approval (Snyder, 1974), and is a widely used concept in social psychology research. High self-monitors 
ADULTHOOD

tend to act in situation-specific ways, whereas low self-monitor individuals show more consistency in their behaviour in social situations. Surprisingly, only one piece of research has reported findings on the role of self-monitoring in preferences for diverse activities, such as listening to pop music (Jamieson, Lydon, \& Zanna, 1987). The results of this study have shown that individuals who score high on self-monitoring are more attracted to others if they share similar activity preferences, such as music. Hargreaves et al. (2006) have noted that social comparison is an important component of musical preferences in childhood and adolescence, as young people try to avoid unpopular music and seek popular music to affiliate themselves with peers. Contrary to conformity and self-monitoring, some adults seek uniqueness and tend to avoid behaviours that are considered normative (Lynn \& Snyder, 2001). Those individuals are attracted to less mainstream attitudes and consumer goods. Although never studied in music, need for uniqueness could partly explain preferences for less mainstream music. The study of interpersonal dispositions broadly speaking is a promising avenue of research into the determinants of musical taste in adulthood that has received almost no recent attention.

In terms of the influence of the social network, research has found that adolescents tend to prefer music genres that are widely accepted by their peer group (Finnäs, 1989; Hargreaves, 1986; Miranda \& Claes, 2009; Mulder et al., 2010; North \& Hargreaves, 1999; Selfhout et al., 2009; van Wel, 1994). More precisely, listening to music in order to be popular, to please friends and to enhance group affiliation have been mentioned as reasons that for listening to particular music styles in adolescence (Bakagiannis \& Tarrant, 2006; Tarrant, North, \& Hargreaves, 2000). Music seems to convey social information that adolescents use to affiliate themselves with specific 
ADULTHOOD

groups of peers (Tarrant et al., 2002). The influence of peers and friends in musical preferences after adolescence is unclear (Bersch-burauel, 2006; Rozin, Riklis, \& Margolis, 2004). Evidence of the influence of family in musical preferences formation in childhood and adolescence has shown that family members, including parents, siblings and children, are surprisingly important (Boer \& Abubakar, 2014; Davidson, Howe, Moore, \& Sloboda, 1996; Finnäs, 1989; Russell, 1997; ter Bogt, Delsing, van Zalk, Christenson, \& Meeus, 2011). Ter Bogt et al. (2011) assessed the transmission of preferences from parents to adolescents. Their results have shown that parental preferences for 'high brow' and rock music were moderate predictors of their children's preferences for the same genres. After adolescence, it seems that the influence of parents in musical taste is common (Bersch-burauel, 2006). As can be seen, the roles of the social network in musical preferences have been examined in childhood and adolescence, and research on those social sources of influences in adults is almost non-existent. In line with the MPAM, the present investigation addresses the issue of the extrinsic sources of influence as determinants of musical preferences in adulthood.

\section{The present research}

To examine interpersonal dispositions and social network as extrinsic determinants of musical preferences in adulthood, we assessed both their direct effects on the musical taste of adults and their moderating effects on age trends in musical preferences.

Broadly speaking, since musical genres labels used in social interactions have been related to stereotyping information that could influence musical preferences (Dunn, de Ruyter, \& Bouwhuis, 2011; Lewis, 1995; Lonsdale \& North, 2009; Rentfrow \& 
ADULTHOOD

Gosling, 2007; Rentfrow, McDonald, \& Oldmeadow, 2009; Ziv, Sagi, \& Basserman, 2008), we hypothesised that preferences for musical genres would be more affected by social influences than preferences for musical clips.

According to Social Investment Theory and related to the social network, adults may be more oriented towards their family and less inclined to seek approval from peers, as compared with adolescents (Roberts, Wood, \& Smith, 2005). Therefore, we hypothesised that adults should be more influenced by their family and less by their peers; this, in turn, should impact their musical preferences.

Interpersonal dispositions might also shape musical taste by defining the degree to which adults are influenced by their social network. Following Leary and Allen's (2011) view of self-presentation, interpersonal dispositions are conceptualised in the present paper as multifaceted and encompassing self-monitoring, conformity, and need for uniqueness. The role of interpersonal dispositions has been seriously overlooked in adulthood. According to the lifespan view of social constraints (Heckhausen, 1998), adults should be disposed to present themselves through music in ways that are aligned with the norms conveyed by their (age-related) social environment.

The present paper looks at these individual differences and social influences variables using the genres and clips subtests of the Music Genre-Clip test (MG-CT), a test of musical preferences that assesses musical genres and clips in parallel (BonnevilleRoussy et al., 2017). The MG-CT uses five musical dimensions of music genres and clips: Classical, Contemporary, Intense, Jazzy, and Unpretentious. Out of these dimensions, three are related to similar dimensions found in the MUSIC model (Contemporary, Intense, and Unpretentious; Bonneville-Roussy et al., 2013; Greenberg et 
ADULTHOOD

al., 2016; Rentfrow et al., 2012, 2011). The two remaining dimensions are thought to be related to the intrinsic musical properties that make them distinct from the other dimensions (Classical and Jazzy; Bonneville-Roussy et al., 2017). Those five dimensions of genres and clips were thoroughly validated in Bonneville-Roussy et al. (2017), and the parallel validity of the dimensions between genres and clips, construct validity, and intermethod reliability have been extensively demonstrated (see Appendix 1, Supplementary Material of Bonneville-Roussy et al., 2017).

In the present research, the social influences variables are examined along with the dimensions of the MG-CT, in samples of adults from 18 to 65 years of age. We examine their unique, main influences on the dimensions, and their interactions with age. In the first study, we examine social influences as determinants of age trends in preferences for musical genres. The second study investigates those determinants using the musical clips subset of the MG-CT. Both studies have received ethical approval from the University of Cambridge Social and Developmental Psychology Ethics Committee.

\section{Study 1 Sources of Social Influences as Determinants of Preferences for Music Genres}

This study explores the associations between social influences and musical preferences using music genres.

\section{Participants, Procedures and Measures}

The sample comprised 656 participants aged between 18 and 64 years old $(M=$ $30.33, S D=10.84)$. They were recruited over the Internet on various crowdsourcing websites between 22 September 2012 and 30 April 2013. Of the participants who 
ADULTHOOD

provided socio-demographic information, $55.6 \%$ were females, and $80.9 \%$ were from white origins. Most of those who mentioned were full time workers (36.1\%) or students $(39.2 \%)$ and the remainder were part-time workers or homemakers. The vast majority of those who mentioned had at least some college or university education (78\%) and five years or less of music education or formal music lessons $(60.2 \%)$.

\section{Measures}

Socio-demographic information, such as age, gender, ethnicity and education were collected.

Music genres. Preferences for music genres were measured with the genres subtest of the MG-CT. The sub-test comprises 17 genres merged into five musical dimensions. The development and validation of this measure is extensively described in Bonneville-Roussy et al. (2017). Participants were asked to rate on a likert-type scale ranging from 1 (I dislike very much) to 5 (I like very much), the extent to which they liked the 17 genres presented to them. Participants also had the opportunity to say if they did not know a genre, in which case the answer was coded as missing data. The musical genres were split into five dimensions, as validated in Bonneville-Roussy et al. (2017). The first genre dimension, which includes classical and opera, was named Classical, reliability Cronbach $\alpha=.66(M=3.37, S D=.94)$. The second genres dimension included ambient/chillout, dance/electro and hip-hop/rap music, and was named Contemporary, $\alpha$ $=.54(M=3.14, S D=.93)$. The Intense genre dimension included heavy metal, punk and rock, $\alpha=.65(M=3.56, S D=.95)$. The fourth genre dimension, Jazzy, includes blues, funk, jazz, Latin and reggae, $\alpha=.72(M=3.26, S D=.75)$. Finally, the Unpretentious genre dimension comprised country, gospel, pop and R\&B/soul, $\alpha=.58(M=3.16, S D$ $=.79)$. 
ADULTHOOD

\section{Sources of Social influences}

Social network. The Social Influences Inventory, which was created for this research, consists of a list of social sources that could influence musical preferences. The items of this survey were created according to the areas of life (e.g. family, friendship, etc.) included in the Quality of Life Inventory (Frisch, Cornell, Villanueva, \& Retzlaff, 1992) that are relevant to musical preferences. The Social Influences Inventory measures, in eight items, the extent to which the musical preferences of participants are influenced by their family, or peers. The general instructions that accompany the inventory are: 'We would like to know about some of the significant sources of influences in your life that might have influenced your musical preferences. Using the scale above, please rate the extent to which these sources were important for your musical taste.' It is measured on a 5-point Likert scale ranging from 1 (not at all) to 5 (a great deal), with an additional 'Not applicable' answer choice provided. Because of the high proportion of missing data for 'Your own children' (approximately $72 \%$ for the two studies), this item was dropped from the analyses. The items comprising the two measures were: Peers (five items: childhood friends, current friends, current or past romantic partners, current or past coworkers, current or past school peers, $M=2.57, S D=.90, \alpha=.76$ ); and Family

Influences (two items: parents, brother and sisters, $M=2.71, S D=1.15, r=.37$ ).

\section{Interpersonal Dispositions}

Self-Monitoring. This questionnaire was measured using the short form of the Self-Monitoring Questionnaire-Revised (Gangestad \& Snyder, 2000; Snyder, 1974). This scale contains 18 items that measure the tendency of individuals to monitor and change 
ADULTHOOD

their self-presentation according to the situation. It is assessed using a true-false (coded 1 and 0, respectively) answer style. Examples of items are: 'I guess I put on a show to impress or entertain others' (demonstrating high self-monitoring) and 'I find it hard to imitate the behavior of other people' (demonstrating low self-monitoring). Scores on the questionnaire were averaged, and the final scores had a theoretical range between 0 (answered all false) and 1 (answered all true). The Self-Monitoring Questionnaire has been used in thousands of studies and has proven to be a reliable and valid measure. The reliability coefficients in the present study reached $\alpha=.73(M=.47, S D=.17)$.

Conformity. The Conformity scale was taken from the International Personality Item Pool (Goldberg et al., 2006). It measures, in five items, the extent to which people worry about the opinion of others and their need to conform to these opinions. It is assessed on a Likert-type scale ranging from 1 (not at all) to 5 (very much). An example of a question is: 'I conform to others' opinions'. The original reliability coefficient was $\alpha=.71$; in relation to this study, the reliability coefficient was $\alpha=.76(M=2.60, S D$ $=.56)$.

Need for uniqueness. The Self-Attributed Need for Uniqueness (SANU) scale is a four-item measure developed by Lynn and Harris (1997) that assesses how different, or unique, individuals want to be as compared with others. It is measured on 5-point Likert scale and an example of a question is: 'Being distinctive is important to me. (1) not at all, (2) slightly, (3) moderately, (4) very, (5) extremely'. In the original validation, the scale achieved good reliability and predictive validity on the propensity to express individuality through consumption. In relation to this study, the coefficient of reliability reached $\alpha=.79(M=2.98$, $S D=.73)$ 
ADULTHOOD

\section{Results}

The direct links between the sources of social influences and musical preferences, were examined using multiple linear regressions. In addition, the moderating effects of social influences on age trends in musical preferences were examined by computing their interaction terms with age, to predict musical preferences. Zero-order correlations are found in Table 1. The following sections deal, first, with the direct influences of age and social influences on the musical preferences of adults, using regression analyses. Second, the moderating effects of each of the social influences are examined, using regression analyses with interaction. The analyses were performed using SPSS 21.

Insert Table 1 about here

Main effects models. Results of the regression analyses, with age and the measures of social network and interpersonal dispositions entered as predictors and the musical dimensions as outcomes, are shown in Table 2. Overall, all the multiple regression analyses of musical preferences on age, interpersonal disposition and social network yield significant results. In the first set of results shown in Table 2, we observe that need for uniqueness positively predicted preferences for Classical music. In terms of the social network, preferences for the Classical genres were positively associated with influences from family members and negatively associated with peer influences. The second set of results presented in Table 2 reveals that the influence of peers positively predicted preferences for the Contemporary music genres and, in terms of interpersonal dispositions, self-monitoring. As indicated in the third section of Table 2, preferences for Intense music was negatively related to conformity dispositions and positively related with the influence of peers. As seen in the fourth section of Table 2, age, need for 
ADULTHOOD

uniqueness, family and peers positively predicted individuals' taste for Jazzy music. The last set of results displayed in Table 2 concerns the Unpretentious music genres dimension that was positively predicted by age, family and peer influences. All in all, the social influences variables yielded small to moderate effect sizes, with multiple $r$ ranging from $r=.18$ to $r=.35$ (Ellis, 2010; Ozer, 1985, 2007).

Insert Table 2 about here

Moderation models. Significant interaction effects are presented in Figure 2.

Using hierarchical multiple regressions separately for each moderating variable, the main effects of standardised age and each of the standardised moderating variables were entered in Step 1, then the interaction term was entered in Step 2. A significant change in $F$ scores at Step 2 would indicate a significant interaction between age and the moderating variables in predicting musical preferences.

Insert Figure 2 about here

As seen in panel A of Figure 2, preferences for the Jazzy genres stayed relatively stable with age for adults low (1 SD below the mean) in Conformity. However, preferences for Jazzy music increased with age for individuals who reported higher levels of conformity ( $1 \mathrm{SD}$ above the mean), $F(3,652)=5.82, p=.001, F_{\text {change }}(1,652)=6.92, p$ $=.009, r=.16$. In panel B of Figure 2, we can observe an interaction between age and conformity to predict Unpretentious genres, $F(3,652)=21.59, p<.001, F_{\text {change }}(1,652)=$ 13.22, $p<.001, r=.30$. Preferences for the Unpretentious genres dimension remained relatively stable with age for the group scoring lower on conformity. A steep positive linear trend was found for individuals scoring higher on conformity: Younger individuals 
ADULTHOOD

who reported more conformity dispositions were also more likely to dislike the Unpretentious genres. On the contrary, middle aged adults who rated themselves high in conformity were also more likely to prefer the Unpretentious genres. No other interaction terms were found, $F_{\text {change }}(1,652)=2.73, p>.10$.

\section{Study 2. Music clips}

In the clips study, we examine the direct and moderating effects of the same sources of social influences on age trends in musical preferences for music clips, using the same methodology as in Study 1.

\section{Participants, Procedures and Measures}

Participants. A total of 301 participants were recruited on the Internet via various mailing lists between February and April 2013. Individuals were aged between 18 and 65 years old $(M=30.92, S D=11.93)$. Of those who stated, $54.8 \%$ were women and $80.1 \%$ were coming from a white ethnic background. They were mostly full time workers $(33.8 \%)$ or students $(45.8 \%)$ and the remainder were part-time workers or homemakers. The vast majority of the sample had at least some college or university education (77.9 $\%)$ and $57.3 \%$ of them had five years or less of formal music lessons.

Procedure. Participants answered online and were first asked to answer a consent form, the music clips included in the MG-CT, and socio-demographic information. To reduce participants' fatigue, participants listened to a random sample of approximately $50 \%$ of the 51 clips available (between 25 and 35 clips). Approximately two weeks later, the participants were invited to answer the social influences questionnaire. 


\section{Measures}

Musical preferences. Musical preferences were measured with the clips sub-test of the MG-CT Short Form (Bonneville-Roussy et al., 2017). The sub-test is comprised of 51 excerpts of music audio-recordings merged into the same 17 genres as in the Genres study (three clips per genre), and then into the Classical $(M=3.38, S D=.93)$, Contemporary $(M=2.69, S D=.80)$, Intense $(M=2.94, S D=.97)$, Jazzy $(M=3.39, S D=$ $.73)$, and Unpretentious $(M=2.69, S D=.72)$ musical clips dimensions. The development and validation of this measure is extensively described in Bonneville-Roussy et al. (2017).

Social network. This measure is described in the Study 1. The reliability coefficients of the dimensions included are: Peers $\alpha=.72(M=2.55, S D=.90)$; and Family $r=.49(M=2.76, S D=1.13)$.

Interpersonal dispositions. In the present study, the reliability coefficient of the Self-Monitoring questionnaire was $\alpha=.74(M=.46, S D=.17)$; the reliability of the Conformity scale was $\alpha=.73(M=2.59, S D=.55)$; finally the reliability of the SANU scale was $\alpha=.74(M=2.95, S D=.74)$. The measures are described in Study 1 .

\section{Results}

Main effects models. The correlations coefficients are found in Table 3. Results of the main effects regression analyses are displayed in Table 4. The multiple regression results of preferences for music clips regressed on age, interpersonal disposition and social network reached significance for only two dimensions: Intense and Unpretentious 
ADULTHOOD

music. In the fourth set of results presented in Table 4, we can see that the Intense dimension negatively predicted by age and positively associated with the influence of peers. Age positively predicted the Unpretentious music clips dimension, as shown in the last section of Table 4. The five models yielded small effect sizes, with multiple correlation coefficients ranging from $r=.17$ to $r=.23$.

Insert Table 3 about here

Moderation models. Significant moderation effects of social influences on age trends in musical preferences are presented in Figure 3. As in Study 1, those differences concern the moderating effects of conformity only, on two musical dimensions:

preferences for the Contemporary and Unpretentious clips dimension. As can be seen in panel A of Figure 3, preferences for the Contemporary music clips stayed relatively stable with age for adults low in conformity. However, preferences for Contemporary music decreased with age for individuals who reported high levels of conformity, $F$ $(3,297)=2.09, p=.10, F_{\text {change }}(1,297)=4.77, p=.03, r=.14$. In cell B of Figure 3 , we can observe an interaction between age and conformity to predict Unpretentious clips, $F$ $(3,297)=6.04, p=.001, F_{\text {change }}(1,297)=9.99, p=.002, r=.24$. Whereas the age trend in preferences for the Unpretentious music clips remained comparatively stable with age for individuals low in conformity, preferences for Unpretentious music, that were below the mean in emerging adulthood, steeply increased with age for individuals high in conformity. No other interaction terms were found $F_{\text {change }}(1,297)<3.15, \mathrm{p}>.07$.

Insert Table 4 about here

\section{Discussion}


ADULTHOOD

This research investigated the two sources of social influences, namely the social network and interpersonal dispositions, as extrinsic determinants of musical preferences in adulthood. The present findings support the overall assumption that musical taste in adulthood is at least partially determined by these sources of social influence. As such, this research advances the literature in the area as it reveals that musical preferences in adulthood can be influenced by contextual (in the present case, social) factors and that adults can be more or less influenced by those factors as they evolve in age.

Insert Figure 3 about here

In line with the MPAM (Bonneville-Roussy et al., 2017), our findings revealed small to medium effects (Cohen, 1988) of age and social influences in preferences for musical genres in adulthood, and small effects in preferences for musical clips. Furthermore, whereas all of the multivariate regression results reached significance in Study 1 (music genres), only two musical dimensions were significant in Study 2 (that is, the Intense and Unpretentious music clips dimensions). These results suggest that preferences for musical genres labelling are influenced by the social network and interpersonal dispositions more than preferences for music audio recordings. That is, musical genres may be filled with extra-musical information such as stereotypes, personality, values, age and gender (see Dunn, de Ruyter, \& Bouwhuis, 2011; Rentfrow, 2012; Shevy, 2008) that may be less salient in the music clips associated with these genres. Interestingly, greater effect sizes were found on the relationship between by age and social influences in preferences for the Unpretentious dimensions, as compared with preferences for the other musical dimensions. This result suggests that social influences 
ADULTHOOD

are more instrumental to the development of taste for some music styles than for others, regardless of the method of measurement.

Interpersonal dispositions were operationalized as encompassing conformity, selfmonitoring and need for uniqueness. Overall, the interpersonal dispositions variables predicted musical genres to a greater extent than musical clips. In addition, the social network, that included the influence of peers (friends and colleagues of roughly the same age), and the family (parents and siblings), was related to almost all of the music genres dimensions, but only one of the music clips dimensions. Those results confirm the aforementioned findings that musical preferences assessed with music genres are more liable to social influences than preferences assessed with audio-recordings (clips).

Our results did not confirm the hypothesis that adults would be more influenced by family than peers. It seems that, in adulthood, some musical dimensions, such as Intense music, are more influenced by peers, whereas other dimensions, such as Classical and Unpretentious music, are more influenced by family members. This finding in aligned with research on the intergenerational transmission of preferences. In individuals for whom family values are important, the intergenerational transmission of cultural resources within the family is more pronounced (Katz-Gerro et al., 2007), and has been associated with family musical habits (Boer \& Abubakar, 2014). Ter Bogt et al. (2011) have also found that the intergenerational transmission of preferences is more noticeable for preferences for Classical music in adolescence. On the contrary, more Intense genres, such as rock, have been associated with a desire to be distinct from their parents and a greater affiliation with peers (Arnett, 1995). Therefore, it seems that, in adolescence, a greater desire to affiliate with family or peers may lead to the adoption of musical 
ADULTHOOD

preferences that are aligned with one or the other group. Our results confirm that the influences of the social network found in adolescence seem to persist in adulthood.

Future research should further examine this hypothesis in adulthood.

All in all, our results suggest that the musical preferences dimensions can be split into three broader categories related to the importance of social influences as extrinsic determinants of musical taste. First, the music genres included in the Classical and Jazzy dimensions are more driven by the need for uniqueness and the direct influences of the social network that are family and peers. In contrast, Intense music, with its more underground music styles, is partially determined by the interpersonal tendency to go against social norms, being negatively related to conformity, and by the influence of peers. Finally, the desire to follow social norms partly shapes preferences for the more mainstream music types (or pop chart music) that are included in the Contemporary and Unpretentious dimensions. One line of research may partly explain the trends found in the present research. According to the reminiscence bump paradigm, middle-aged adults should more accurately remember and like the pop music they listened to when they were young adults, because this music should be better encoded and charged with positive emotions (Krumhansl \& Zupnick, 2013). The reminiscence bump hypothesis needs to be investigated further in relation to social influences and preferences for various musical style.

We found one consistent finding between the musical genres and clips dimensions of preferences with regards to the moderating effects of social influences on age trends in musical preferences: Conformity was the only variable that moderated the links between age and musical preferences. Conformity was conceptualised as the psychological 
tendency to conform to the social norms that are salient in one's social group (Cialdini \& Trost, 1998). According to the media effect, frequent contact with some types of consumer goods advertised in the media (such as music) lead people to act in a way that is consistent with the values and behavior associated with those goods (see Rentfrow, 2012). Moreover, individuals who are more prone to conformity are more likely to conform to the taste of others and to avoid attitudes and behaviors that are perceived negatively by others. Research has shown that when the choice of goods or brands is more socially visible (such as preference for a music genre), this choice is more vulnerable to the influences of age-related norms than when this choice is less visible (such as preference for a specific song; Mandrik, Fern, \& Bao, 2005). Research on music and stereotyping indicates that the different musical dimensions are related to agespecific stereotypes that might also influence musical taste with age (Lewis, 1975). On a side note, our results replicated the results of past research that conformity propensity decreases with age (Costanzo \& Shaw, 1966; Lodi-Smith \& Roberts, 2010; Pasupathi, 1999).

Conformity was a significant moderator of the age trends of Unpretentious music both for genres and clips. Being a middle-aged adult comes with responsibilities and a social status that might be incompatible with stereotypes and social norms associated with some music styles (such as Intense with genres such as heavy metal), but more compatible with the styles associated with the Unpretentious dimension (with genres such as country) (North \& Fiske, 2013). Furthermore, middle-aged adults are aware of agerelated social norms and show concern for the appropriateness of their own behaviour 
ADULTHOOD

(Heckhausen, 1998). The Unpretentious music styles might convey stereotypes and values that are more aligned with the concerns of middle-aged adults.

According to Heckhausen (1998), as individuals age, and although individuals tend to rate themselves as less conformist with age, more and more social constraints are put on normative behaviour that might put pressure on individuals to change their taste according to the social norms of their age groups. Interestingly, individuals who rated themselves higher in conformity also tended to prefer genres that were aligned with their age groups, thus confirming Heckhausen's (1998) hypothesis (e.g. Unpretentious for middle-aged adults and Contemporary for emerging adults, see Bonneville-Roussy et al., 2013, 2017). This tendency was less marked for individuals low on conformity, who tended to display more constant musical preferences with age. In addition, according to Carstensen (1995), and Charles and Carstensen (2010), older people reduce their number acquaintances to only a few emotionally close peers. As such, older adults may be less influenced by the music of their peers because the number of peers is significantly reduced. It is also possible that music has a much weaker social function in middle adulthood than in early adulthood (Schäfer, 2016).

On a more general note, our results reveal that the musical taste of adults is influenced by their own social network and their interpersonal disposition. Past research has shown that Contemporary and Intense music might be more appropriate in adolescence and emerging adulthood, whereas Unpretentious, Mellow and Jazzy music is preferred by the older adult age groups (Bonneville-Roussy et al., 2013, 2017). The social influences trends found in the present research support this finding. Emerging adults who were particularly concerned with their self-presentation might have tended to prefer the 
ADULTHOOD

Contemporary genres because this type of music may be more widely accepted in emerging adulthood. On the contrary, Unpretentious music genres seem to convey stereotypes associated with traditional values and conservatism (Lewis, 1995), values that may be endorsed later in adulthood (Tilley \& Evans, 2014). In addition, Unpretentious music has been associated with the social norms of a more mature social sub-culture (North \& Hargreaves, 2007a; Shevy, 2008) and could be generally more attractive to middle-aged adults. Therefore, it is not surprising to see that the Unpretentious music genres were mostly liked by middle adults who had stronger conformity dispositions. Overall, the present findings suggest that the age-related social constraints hypothesised by Heckhausen (1998) can effectively be applied to musical preferences.

\section{Conclusion}

This research is the first to give an account of the relations between age, social influences, and musical preferences in adulthood. In line with the MPAM vision of musical taste in adulthood, the effects of social influences and interpersonal dispositions, conceptualised in the MPAM as extrinsic determinants of musical taste, seem to have been at least equal or even greater than the effects of the other type of extrinsic determinants reported in past research (Bonneville-Roussy et al., 2013; Langmeyer et al., 2012; Rentfrow \& Gosling, 2003; Zweigenhaft, 2008). The present research shows that conformity seems particularly important in explaining age differences in preferences in adulthood. These results suggest that music may also serve as a "badge for social identity” (North \& Hargreaves, 1999) beyond adolescence and throughout adulthood. 
ADULTHOOD

The research presented here offers a snapshot of the role of social influences in changes in musical preferences in adulthood that would be best confirmed by longitudinal studies spanning decades. The latter type of study design requires resources that are not easily accessible to music psychologists, therefore, cross-sequential studies, looking at two or three cohorts of adults for a limited time span, might be a good alternative solution to pure longitudinal studies. Clearly, more research is needed to investigate the relations between the extrinsic determinants of preferences, especially social influences and conformity, and age trends in musical preferences throughout the lifespan. 


\section{References}

Arnett, J. J. (1992). The soundtrack of recklessness: Musical preferences and reckless behavior among adolescents. Journal of Adolescent Research, 7, 313-331.

Arnett, J. J. (1995). Adolescents' uses of media for self-socialization. Journal of Youth and Adolescence, 24, 519-533.

Bakagiannis, S., \& Tarrant, M. (2006). Can music bring people together? Effects of shared musical preference on intergroup bias in adolescence. Scandinavian Journal of Psychology, 47, 129-36.

Bearden, W. O., \& Rose, R. L. (1990). Attention to social comparison information: An individual difference factor affecting consumer conformity. Journal of Consumer Research, 16, 461.

Bennett, A., \& Taylor, J. (2012). Popular music and the aesthetics of ageing. Popular Music, 31, 231-243.

Berger, J., \& Heath, C. (2008). Who drives divergence? Identity signaling, outgroup dissimilarity, and the abandonment of cultural tastes. Journal of Personality and Social Psychology, 95, 593-607.

Berns, G. S., Capra, C. M., Moore, S., \& Noussair, C. (2010). Neural mechanisms of the influence of popularity on adolescent ratings of music. NeuroImage, 49, 2687-96.

Bersch-burauel, A. (2006). Development of musical preferences in adulthood.

Proceedings of the 9th International Conference on Music Perception and Cognition (pp. 1607-1611). Bologna, Italy.

Boer, D., \& Abubakar, A. (2014). Music listening in families and peer groups: Benefits for young people's social cohesion and emotional well-being across four cultures. 
ADULTHOOD

Frontiers in Psychology, 5(392). doi:10.3389/fpsyg.2014.00392

Boer, D., Fischer, R., Strack, M., Bond, M. H., Lo, E., \& Lam, J. (2011). How shared preferences in music create bonds between people: Values as the missing link. Personality and Social Psychology Bulletin, 37, 1159-1171.

Bonneville-Roussy, A., Rentfrow, P. J., Xu, M. K., \& Potter, J. (2013). Music through the ages: Trends in musical engagement and preferences from adolescence through middle adulthood. Journal of Personality and Social Psychology, 105, 703-17.

Bonneville-Roussy, A., Stillwell, D., Kosinski, M., \& Rust, J. (2017). Age trends in musical preferences in adulthood: 1 . Conceptualization and empirical investigation. Musicae Scientiae. Advance online publication. doi:10.1177/1029864917691571

Brown, R. A. (2012). Music preferences and personality among Japanese university students. International Journal of Psychology: Journal International de Psychologie, 47, 259-68.

Buss, D. M., \& Craik, K. H. (1981). The act frequency analysis of interpersonal dispositions: Aloofness, gregariousness, dominance and submissiveness. Journal of Personality, 49, 175-192.

Carstensen, L. L. (1995). Evidence for a life-span theory of socioemotional selectivity. Current Directions in Psychological Science, 4, 151-156.

Chamorro-Premuzic, T., Goma -i-Freixanet, M., Furnham, A., Muro, A., \& Gomà-iFreixanet, M. (2009). Personality, self-estimated intelligence, and uses of music: A Spanish replication and extension using structural equation modeling. Psychology of Aesthetics, Creativity, and the Arts, 3, 149-155.

Chamorro-Premuzic, T., Swami, V., Furnham, A., \& Maakip, I. (2009). The Big Five 
ADULTHOOD

personality traits and uses of music. Journal of Individual Differences, 30, 20-27.

Charles, S. T., \& Carstensen, L. L. (2010). Social and emotional aging. Annual Review of Psychology, 61, 383-409.

Cialdini, R., \& Trost, M. (1998). Social influence: Social norms, conformity and compliance. In D. T. Gilbert, S. T. Fiske, \& G. Lindzey (Eds.), The handbook of social psychology (4th ed., pp. 151-192). New York: McGraw-Hill.

Cohen, J. (1988). Statistical power analysis for the behavioral sciences.

Colley, A. (2008). Young people's musical taste: Relationship with gender and genderrelated traits. Journal of Applied Social Psychology, 38, 2039-2055.

Costanzo, P. R., \& Shaw, M. E. (1966). Conformity as a function of age level. Child Development, 37, 967-975.

Davidson, J. W., Howe, M. J. a., Moore, D. G., \& Sloboda, J. a. (1996). The role of parental influences in the development of musical performance. British Journal of Developmental Psychology, 14, 399-412.

Delsing, M. J. M. H., ter Bogt, T. F. M., Engels, R. C. M. E., \& Meeus, W. H. J. (2008). Adolescents' music preferences and personality characteristics. European Journal of Personality, 22, 109-130.

Dunn, G. P., de Ruyter, B., \& Bouwhuis, D. G. (2011). Toward a better understanding of the relation between music preference, listening behavior, and personality. Psychology of Music, 40, 411-428.

Egermann, H., Kopiez, R., \& Altenmüller, E. (2013). The influence of social normative and informational feedback on musically induced emotions in an online music listening setting. Psychomusicology: Music, Mind, and Brain, 23, 21-32. 


\section{ADULTHOOD}

Ellis, P. D. (2010). The essential guide to effect sizes: Statistical power, meta-analysis, and the interpretation of research results. Cambridge: Cambridge University Press.

Finnäs, L. (1989). How can musical preferences be modified? A research review. Council for Research in Music Education, 102, 1-58.

Frisch, M. B., Cornell, J., Villanueva, M., \& Retzlaff, P. J. (1992). Clinical validation of the Quality of Life Inventory. A measure of life satisfaction for use in treatment planning and outcome assessment. Psychological Assessment, 4, 92-101.

Gangestad, S. W., \& Snyder, M. (2000). Self-monitoring: Appraisal and reappraisal. Psychological Bulletin, 126, 530-555.

Getz, L. M., Chamorro-Premuzic, T., Roy, M., \& Devroop, K. (2011). The relationship between affect, uses of music, and music preferences in a sample of South African adolescents. Psychology of Music, 40, 164-178.

Goldberg, L. R., Johnson, J. a., Eber, H. W., Hogan, R., Ashton, M. C., Cloninger, C. R., \& Gough, H. G. (2006). The international personality item pool and the future of public-domain personality measures. Journal of Research in Personality, 40, 84-96.

Greasley, A. E., \& Lamont, A. M. (2006). Music preference in adulthood : Why do we like the music we do ? Proceedongs of the 9th International Conference on Music Perception and Cognition. Bologna, Italy.

Greenberg, D. M., Kosinski, M., Stillwell, D. J., Monteiro, B. L., Levitin, D. J., \& Rentfrow, P. J. (2016). The Song Is You: Preferences for musical attribute dimensions reflect personality. Social Psychological and Personality Science, 7, $597-605$.

Greenberg, D. M., Monteiro, B. L., \& Rentfrow, P. J. (2014). External correlates of 


\section{ADULTHOOD}

musical preferences: A meta-analysis. Manuscript in Preparation.

Han, S.-K. (2003). Unraveling the brow: What and how of choice in musical preference. Sociological Perspectives, 46, 435-459.

Hargreaves, D. J. (1986). The developmental psychology of music. Cambridge, UK: Cambridge University Press.

Hargreaves, D. J., \& Bonneville-Roussy, A. (2017). What is "open-earedness", and how can it be measured? Musicae Scientiae. Advance online publication. doi:10.1177/1029864917697783.

Hargreaves, D. J., Comber, C., \& Colley, A. (1995). Effects of age, gender, and training on musical preferences of British secondary school students. Journal of Research in Music Education, 43, 242.

Hargreaves, D. J., North, A. C., \& Tarrant, M. (2006). Musical preference and taste in childhood and adolescence. In G. E. McPherson (Ed.), The child as musician: $A$ handbook of musical development (pp. 135-154). Oxford: Oxford University Press.

Harrison, J., \& Ryan, J. (2010). Musical taste and ageing. Ageing and Society, 30, 649669.

Hays, T., \& Minichiello, V. (2005). The meaning of music in the lives of older people: A qualitative study. Psychology of Music, 33, 437-451.

He, L., Cong, F., Liu, Y., \& Zhou, X. (2010). The pursuit of optimal distinctiveness and consumer preferences. Scandinavian Journal of Psychology, 411-417.

Heckhausen, J. (1998). Developmental Regulation in Adulthood. Cambridge: Cambridge University Press.

Hemming, J. (2013). Is there a peak in popular music preference at a certain songspecific age? A replication of Holbrook \& Schindler's 1989 study. Musicae 
ADULTHOOD

Scientiae, 17, 293-304.

Jamieson, D. W., Lydon, J. E., \& Zanna, M. P. (1987). Attitude and activity preference similarity: Differential bases of interpersonal attraction for low and high selfmonitors. Journal of Personality and Social Psychology, 53, 1052-1060.

Katz-Gerro, T. (1999). Cultural consumption and social stratification: Leisure activities, musical tastes, and social location. Sociological Perspectives, 42, 627-646.

Katz-Gerro, T., Raz, S., \& Yaish, M. (2007). Class, status, and the intergenerational transmission of musical tastes in Israel. Poetics, 35, 152-167.

Knox, D., \& MacDonald, R. (2016). Broadcasting personalities: The relationship between occupation and music preferences in the BBC Radio programme Desert Island Discs. Psychology of Music. Advance online ublication. doi:10.1177/0305735616670497

Krumhansl, C. L., \& Zupnick, J. A. (2013). Cascading reminiscence bumps in popular music. Psychological Science, 24, 2057-68.

Langmeyer, A., Guglhör-Rudan, A., \& Tarnai, C. (2012). What do music preferences reveal about personality? Journal of Individual Differences, 33, 119-130.

Leary, M. R., \& A llen, A. B. (2011). Self-presentational persona: Simultaneous management of multiple impressions. Journal of Personality and Social Psychology, $101,1033-49$.

Lewis, G. H. (1975). Cultural socialization and the development of taste cultures and culture classes in American popular music: Existing evidence and proposed research directions 1. Popular Music and Society, 4, 226-241.

Lewis, G. H. (1995). Taste cultures and musical stereotypes: Mirrors of identity? Popular Music and Society, 19, 37-58. 
ADULTHOOD

Lodi-Smith, J., \& Roberts, B. W. (2010). Getting to know me: Social role experiences and age differences in self-concept clarity during adulthood. Journal of Personality, $78,1383-410$.

Lonsdale, A. J., \& North, A. C. (2009). Musical taste and ingroup favouritism. Group Processes \& Intergroup Relations, 12, 319-327.

Lynn, M., \& Harris, J. (1997). Individual differences in the pursuit of self-uniqueness through consumption. Journal of Applied Social Psychology, 27, 1861-1883.

Lynn, M., \& Snyder, M. (2001). Uniqueness seeking. In M. Snyder \& S. Lopez (Eds.), Handbook of positive psychology (pp. 395-410). New York: Oxford University Press.

Macdonald, R., Hargreaves, D. J., \& Miell, D. (2002). Musical Identities. New York: Oxford University Press.

Mandrik, C. a., Fern, E. F., \& Bao, Y. (2005). Intergenerational influence: Roles of conformity to peers and communication effectiveness. Psychology and Marketing, $22,813-832$.

Mark, N. (1998). Birds of a feather sing together. Social Forces, 77, 453-485.

Martin, K. A., Leary, M. R., \& Rejeski, W. J. (2000). Self-presentational concerns in older adults: Implications for health and well-being. Basic and Applied Social Psychology, 22, 169-179.

McCown, W., Keiser, R., Mulhearn, S., \& Williamson, D. (1997). The role of personality and gender in preference for exaggerated bass in music. Personality and Individual Differences, 23, 543-547.

Miranda, D., Blais-Rochette, C., Vaugon, K., Osman, M., \& Arias-Valenzuela, M. 
ADULTHOOD

(2015). Towards a cultural-developmental psychology of music in adolescence. Psychology of Music, 43(2), 197-218.

Miranda, D., \& Claes, M. (2004). Rap music genres and deviant behaviors in FrenchCanadian adolescents. Journal of Youth and Adolescence, 33, 113-122.

Miranda, D., \& Claes, M. (2009). Music listening, coping, peer affiliation and depression in adolescence. Psychology of Music, 37, 215-233.

Mulder, J., Ter Bogt, T. F. M., Raaijmakers, Q. A. W., Nic Gabhainn, S., \& Sikkema, P. (2009). From death metal to R\&B? Consistency of music preferences among Dutch adolescents and young adults. Psychology of Music, 38, 67-83.

Mulder, J., Ter Bogt, T. F. M., Raaijmakers, Q. a W., Nic Gabhainn, S., Monshouwer, K., \& Vollebergh, W. a M. (2010). Is it the music? Peer substance use as a mediator of the link between music preferences and adolescent substance use. Journal of Adolescence, 33, 387-94.

Nater, U. M., Abbruzzese, E., Krebs, M., \& Ehlert, U. (2006). Sex differences in emotional and psychophysiological responses to musical stimuli. International Journal of Psychophysiology: Official Journal of the International Organization of Psychophysiology, 62, 300-8.

North, A. C. (2010). Individual differences in musical taste. American Journal of Psychology, 123, 199-208.

North, A. C., \& Hargreaves, D. J. (1999). Music and adolescent identity. Music Education Research, 1, 75-92.

North, A. C., \& Hargreaves, D. J. (2007a). Lifestyle correlates of musical preference: 1. Relationships, living arrangements, beliefs, and crime. Psychology of Music, 35, 58- 


\section{ADULTHOOD}

87.

North, A. C., \& Hargreaves, D. J. (2007b). Lifestyle correlates of musical preference: 2. Media, leisure time and music. Psychology of Music, 35, 179-200.

North, A. C., \& Hargreaves, D. J. (2007c). Lifestyle correlates of musical preference: 3. Travel, money, education, employment and health. Psychology of Music, 35, 473497.

North, A. C., \& Hargreaves, D. J. (2008a). Musical preference and taste. In A.C. North \& D. J. Hargreaves (Eds.), The social and applied psychology of music (pp. 75-142). Oxford: Oxford University Press.

North, A. C., \& Hargreaves, D. J. (Eds.). (2008b). The social and applied psychology of music. Oxford: Oxford University Press.

North, M. S., \& Fiske, S. T. (2013). Act your (old) age: Prescriptive, ageist biases over succession, consumption, and identity. Personality \& Social Psychology Bulletin, $39,720-34$.

Ozer, D. J. (1985). Correlation and the coefficient of determination. Psychological Bulletin, 97, 307-315.

Ozer, D. J. (2007). Evaluating effect size in personality research. In R. W. Robins, R. C. Fraley, \& R. F. Krueger (Eds.), Handbook of research methods in personality psychology (pp. 495-501). New York: Guilford Press.

Pasupathi, M. (1999). Age differences in response to conformity pressure for emotional and nonemotional material. Psychology and Aging, 14, 170-174.

Rentfrow, P. J. (2012). The role of music in everyday life: Current directions in the social psychology of music. Social and Personality Psychology Compass, 6, 402-416. 
ADULTHOOD

Rentfrow, P. J., Goldberg, L. R., \& Levitin, D. J. (2011). The structure of musical preferences: a five-factor model. Journal of Personality and Social Psychology, 100, $1139-57$.

Rentfrow, P. J., Goldberg, L., Stillwell, D., Kosinski, M., Gosling, S. D., \& Levitin, D. J. (2012). The song remains the same: A replication and extension of the MUSIC model. Music Perception, 30, 161-185.

Rentfrow, P. J., \& Gosling, S. D. (2006). Message in a ballad: The role of music preferences in interpersonal perception. Psychological Science, 17, 236-242.

Rentfrow, P. J., \& Gosling, S. D. (2007). The content and validity of music-genre stereotypes among college students. Psychology of Music, 35, 306-326.

Rentfrow, P. J., \& McDonald, J. A. (2009). Music preferences and personality. In P. Juslin \& J. A. Sloboda (Eds.), Handbook of music and emotion (pp. 669-695). Oxford: Oxford University Press.

Rentfrow, P. J., McDonald, J. A., \& Oldmeadow, J. A. (2009). You are what you listen to: Young people's stereotypes about music fans. Group Processes \& Intergroup Relations, 12, 329-344.

Roberts, B. W., Wood, D., \& Smith, J. L. (2005). Evaluating Five Factor Theory and social investment perspectives on personality trait development. Journal of Research in Personality, 39, 166-184.

Rozin, P., Riklis, J., \& Margolis, L. (2004). Mutual exposure or close peer relationships do not seem to foster increased similarity in food, music or television program preferences. Appetite, 42, 41-8.

Russell, P. A. (1997). Musical tastes and society. In D. J. Hargreaves \& A. C. North 
ADULTHOOD

(Eds.), The social psychology of music (pp. 141-158). New York, NY: Oxford University Press.

Schäfer, T. (2016). The goals and effects of music listening and their relationship to the strength of music preference. PLoS ONE, 11, e0151634.

doi:10.1371/journal.pone.0151634

Schäfer, T., Auerswald, F., Bajorat, I. K., Ergemlidze, N., Frille, K., Gehrigk, J., ... Wilker, T. (2016). The effect of social feedback on music preference. Musicae Scientiae, 20, 263-268.

Schäfer, T., \& Sedlmeier, P. (2010). What makes us like music? Determinants of music preference. Psychology of Aesthetics, Creativity, and the Arts, 4, 223-234.

Schwartz, K. D., \& Fouts, G. T. (2003). Music preferences, personality style, and developmental issues of adolescents. Journal of Youth and Adolescence, 32, 205213.

Selfhout, M. H. W., Branje, S. J. T., ter Bogt, T. F. M., \& Meeus, W. H. J. (2009). The role of music preferences in early adolescents' friendship formation and stability. Journal of Adolescence, 32, 95-107.

Shevy, M. (2008). Music genre as cognitive schema: extramusical associations with country and hip-hop music. Psychology of Music, 36, 477-498.

Snyder, M. (1974). Self-monitoring of expressive behavior. Journal of Personality and Social Psychology, 30, 526-537.

Tarrant, M., North, A. C., \& Hargreaves, D. J. (2000). English and American adolescents' reasons for listening to music. Psychology of Music, 28, 166-173.

ter Bogt, T. F. M., Delsing, M. J. M. H., van Zalk, M., Christenson, P. G., \& Meeus, W. 
ADULTHOOD

H. J. (2011). Intergenerational continuity of taste: Parental and adolescent music preferences. Social Forces, 90, 297-319.

ter Bogt, T. F. M., Gabhainn, S. N., Simons-Morton, B. G., Ferreira, M., Hublet, A., Godeau, E., ... Richter, M. (2012). Dance is the new metal: adolescent music preferences and substance use across Europe. Substance Use \& Misuse, 47, 130-42.

Ter Bogt, T. F. M., Mulder, J., Raaijmakers, Q. a. W., \& Nic Gabhainn, S. (2010). Moved by music: A typology of music listeners. Psychology of Music, 39, 147-163.

Tilley, J., \& Evans, G. (2014). Ageing and generational effects on vote choice:

Combining cross-sectional and panel data to estimate APC effects. Electoral Studies, 33, 19-27.

van Eijck, K. (2001). Social differentiation in musical taste patterns. Social Forces, 79, $1163-1185$.

van Wel, F. (1994). A culture gap between the generations? Social influences on youth cultural style. International Journal of Adolescence and Youth, 4, 211-228.

Ziv, N. (2016). Music and compliance: Can good music make us do bad things? Psychology of Music, 44(5), 953-966.

Ziv, N., Sagi, G., \& Basserman, K. (2008). The effect of looks and musical preference on trait inference. Psychology of Music, 36, 463-476.

Zweigenhaft, R. L. (2008). A Do Re Mi Encore. Journal of Individual Differences, 29, $45-55$. 